\title{
What Is Causing Vomiting in a 5-Month-Old Infant?
}

\author{
John W. Harrington, MD
}

A 5-month-old infant was brought to the emergency department (ED) with vomiting and concerns for reflux. He was a full-term infant born by $\mathrm{C}$-section due to failure to progress, with a birth weight of $3.3 \mathrm{~kg}$. He had an uneventful nursery stay at the time of birth and has been to all well visits at his pediatrician's office. The patient has been gaining appropriate weight.

\section{Physical examination}

In the ED, he did not appear dehydrated and had a normal capillary refill of less than $2 \mathrm{sec}$, a normal heart rate, and normal moist mucous membranes. The infant was given ondansetron and tolerated $3 \mathrm{oz}$ of formula.

His mother was concerned about pyloric stenosis, since it was diagnosed in her nephew at the same age as her son. Therefore, the attending physician in the ED performed an ultrasonography scan, results of which showed a normal pylorus. The infant was then discharged home.

The following day, the infant and his mother followed up with the general pediatrician. The patient's mother was still concerned, since the infant continued to vomit/reflux with no fever or diarrhea, has only had 2 wet diapers since the morning, and seemed a little tired.

The pediatrician tried an oral feeding in the office, and the infant promptly vomited. The infant also had a slightly raised, bumpy rash with some reddish/ purplish discoloration that blanch. The pediatrician administered a dose of ondansetron, and the infant tolerated 3 to 4 oz of formula.

The mother felt that the infant looked much better after the feeding and took the infant home. Overnight, the infant was brought to the ED again with the same concern of vomiting/reflux and appeared mildly dehydrated.

\section{At this point, what differential diagno-} ses do you consider?
A. Hiatal Hernia
B. Acute Gastroenteritis
C. Gastroesophageal reflux
D. None of the above

\section{Answer: D. None of the above}

\section{AFFILIATIONS:}

Director, Division of General Academic Pediatrics, Children's Hospital of The King's Daughters, Norfolk, VA CITATION:

Harrington JW. What caused vomiting in a 5-month-old infant? Consultant. Published online May 26, 2021. doi:10.25270/con.2021.05.00011

Received May 12, 2021. Accepted May 14, 2021.

\section{DISCLOSURES:}

The authors report no relevant financial relationships.

\section{CORRESPONDENCE:}

John W. Harrington, MD, 601 Children's Lane, Norfolk, VA 23507 (John.Harrington@chkd.org)

Although answer $A$ is not a bad thought, it does not broaden the differential as much as it should. Some clinicians find it useful to use the mnemonic of VINDICATE to help expand their differential.' It stands for:

- Vascular

- Infectious

- Neoplastic

- Degenerative

- latrogenic/Intoxication

- Congenital

- Autoimmune

- Trauma

- Endocrine/Metabolic

A chart review showed that he had had a newborn screen within the normal limits at the time of birth. At the time of this visit, the infant had a normal level of electrolytes, and no metabolic acidosis. His complete blood cell count had showed a slightly decreased level of hemoglobin, at $9.3 \mathrm{~g} / \mathrm{dL}$, and a white blood cell count within normal limits. He had an unremarkable urinalysis, with blood and urine cultures obtained. A polymerase chain reaction test via nasal swab was conducted to evaluate for multiple viruses, results of which were negative. His pro-time and prothrombin time were within normal limits. However, he had some mildly elevated liver enzymes, with an aspartate transaminase level of $163 \mathrm{U} / \mathrm{L}$ (reference range, 35-140 U/L) and alanine aminotransferase level of $93 \mathrm{U} / \mathrm{L}$ (reference range, 12-45 U/L).

An intravenous line was initiated to administer normal saline to the infant. Immediately after initiation, he developed a brief 3-minute seizure. 


\section{What would you do next?}
A. Urine Toxicology
B. Call Neurology
C. EEG (Electroencephalogram)
D. Neuroimaging
E. All the above

\section{Answer: E. All of the above}

An initial computed tomography (CT) scan without contrast of the head was performed, results of which showed bilateral subdural hematomas. The next day, a magnetic resonance imaging (MRI) scan was performed (Figures 1 and 2). Parental history was revised to state that the infant had rolled off a couch, about 18 inches in height, 3 days prior to presentation and may have bumped his head on the hardwood floor. The patient was in the care of his father during the time of the fall. Additional studies showed a normal skeletal survey, but a dilated fundoscopic examination showed multiple retinal hemorrhages. The case was referred to child protective services and is still under review.

\section{Discussion}

This pediatric case highlights the need to be vigilant in terms of questioning your diagnostic thought process instead of anchoring to one diagnosis or focusing on one symptom. This firstlevel, quick thinking is one explanation for diagnostic delays that can be managed by the provider. The infant had never developed a fever or diarrhea, and generally reflux starts before 5 months of age, so the reason for vomiting needed further evaluation and an expansion of the original differential.

There have been validated concerns that the pandemic is putting additional stress on families and increasing the risk of child abuse and neglect. ${ }^{2}$ This concern has been difficult to measure due to the precipitous drop in visits to the ED and reports of abuse, because schools and child centers have been closed, and this is where many of the reports are generated. In 2 single center studies from

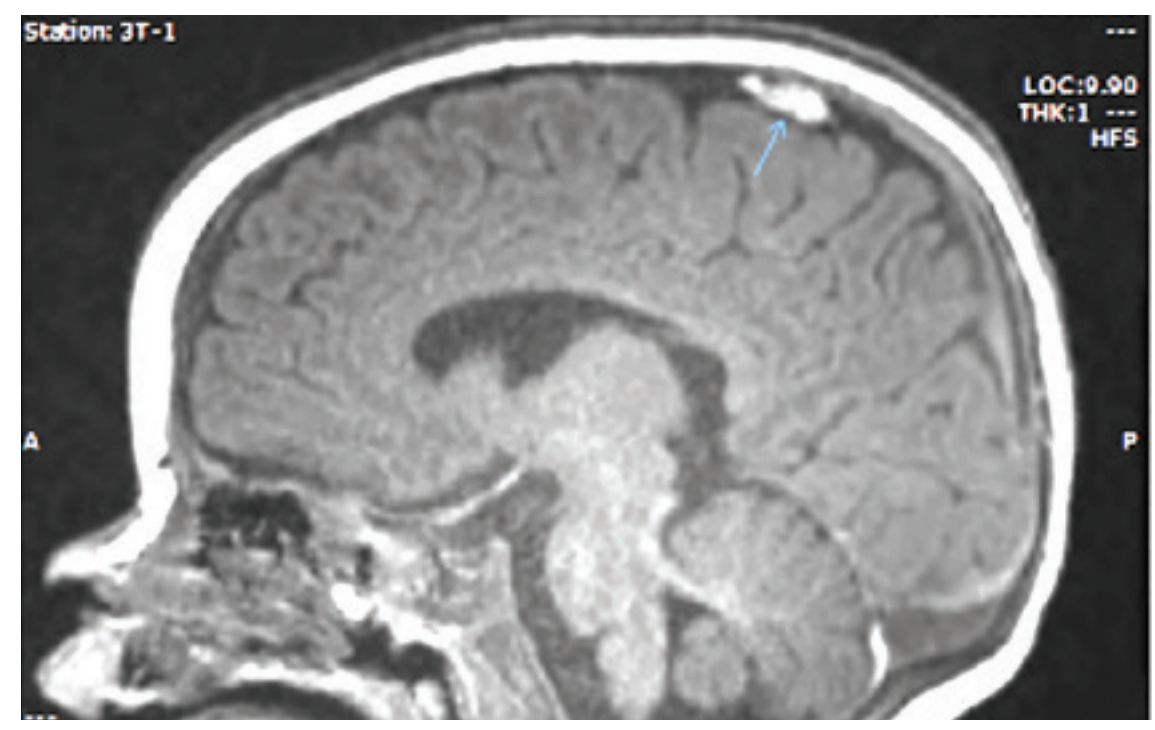

Figure 1. MRI scan with the focal subdural on high left and right parasagittal apices.

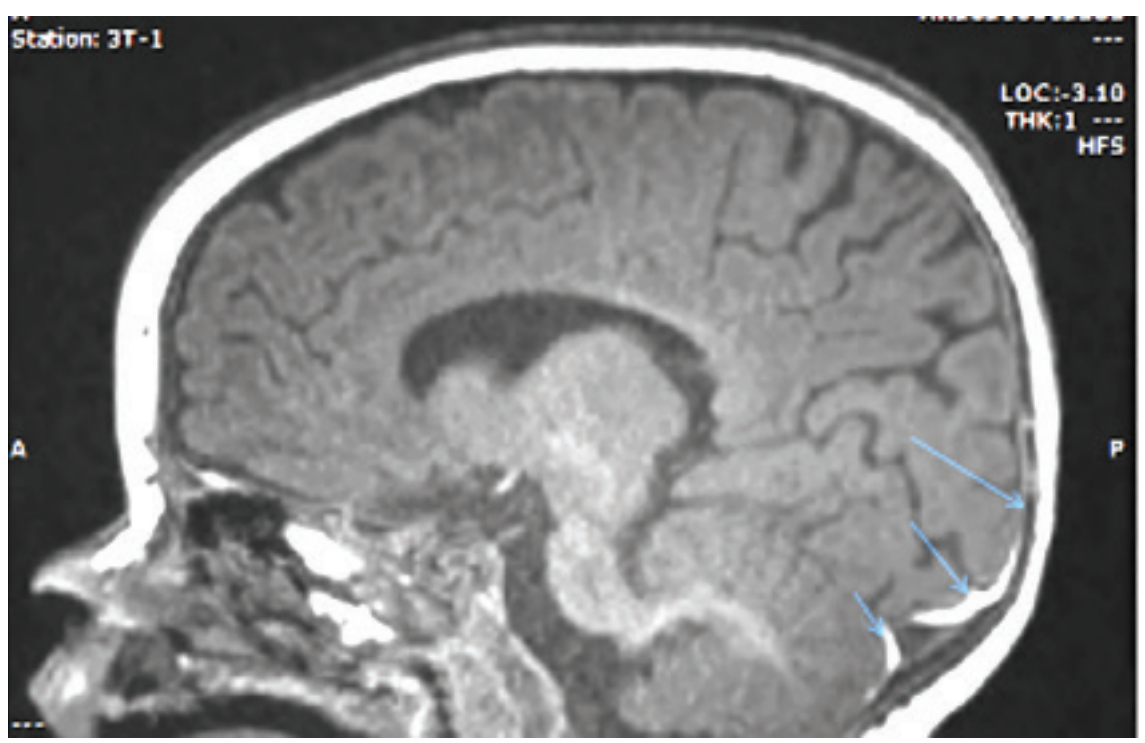

Figure 2. MRI scan with layering subdural in the occipital region.

2020 that examined visits for trauma, there did not appear to be an increase in incidents of unexpected trauma, but this may only confirm the decrease in volume of patients presenting to the ED versus an actual decrease..$^{3,4}$

This conundrum is examined further in a report from the Centers for Disease Control and Prevention that states there has been a shift in the percentage of trauma cases per 100,000 upward for abuse, and the actual number of cases has remained steady. ${ }^{2}$ It is also possible that fewer people with abusive injuries that were not life-threatening did not make it to the ED because of the stay-athome orders during the pandemic, and this could be a reason why no increase has been seen. Overall, child abuse and nonaccidental trauma must always be on one's differential when dealing with the pediatric population. 
Data from 2019 as reported by the US Children's Bureau show that an estimated 656,000 children had experienced abuse and neglect, and about $84.5 \%$ of these children had experienced a single type of maltreatment. ${ }^{5}$ Parsing this out into the collected categories, it is estimated that about $61 \%$ had experienced neglect, $10 \%$ had experienced physical abuse, and $7 \%$ had experienced sexual abuse. The report also states that more than $15 \%$ of children had experienced 2 or more types of maltreatment. ${ }^{5}$

Most workups for physical abuse in children aged 2 years or younger advocate for a skeletal survey. ${ }^{6} \mathrm{~A}$ recent study utilizing the Pediatric Hospital Information System database shows that hospital systems utilizing a child abuse clinical pathway for children with injuries associated with a high risk of abuse had greater odds of receiving a skeletal survey. ${ }^{7}$ Interestingly, there were higher rates of skeletal surveys performed on infants with public vs private insurance regardless of a pathway. ${ }^{7}$

Children with abusive head trauma should also have an ophthalmic evaluation to further clarify the injury as abusive vs accidental. ${ }^{8}$ In our patient, there were multiple retinal hemorrhages, which made the likelihood of a fall off an 18-inch-tall couch less likely and abusive head trauma more likely. As stated earlier, a child protective services representative is investigating the decision to enter the child into foster care.

\section{References}

1. VINDICATE. Segen's Medical Dictionary, 2011. Accessed May 18, 2021. https://medical-dictionary.thefreedictionary.com/VINDICATE

2. Swedo $E$, Idaikkadar $N$, Leemis $R$, et al. Trends in U.S. emergency department visits related to suspected or confirmed child abuse and neglect among children and adolescents aged $<18$ years before and during the COVID-19 pandemic - United States, January 2019-September 2020. MMWR Morb Mortal Wkly Rep. 2020;69(49):18411847. http://dx.doi.org/10.15585/mmwr. mm6949a1

3. Sherman WF, Khadra HS, Kale NN, Wu VJ, Gladden PB, Lee OC. how did the number and type of injuries in patients presenting to a regional level $\mathrm{i}$ trauma center change during the COVID-19 pandemic with a stay-at-home order? Clin Orthop Relat Res. 2021;479(2):266-275. https://doi. org/10.1097/corr.0000000000001484
4. Murphy $\mathrm{T}$, Akehurst $\mathrm{H}$, Mutimer J. Impact of the 2020 COVID-19 pandemic on the workload of the orthopaedic service in a busy UK district general hospital. Injury, 2020;51(10):2142-2147. https://doi. org/10.1016/j.injury.2020.07.001

5. Child maltreatment 2019. US Department of Health \& Human Services, Administration for Children and Families, Administration on Children, Youth and Families, and Families Children's Bureau. Published 2021. Accessed May 18, 2021. https://www.acf. hhs.gov/sites/default/files/documents/cb/ cm2019.pdf

6. Expert Panel on Pediatric Imaging; Wootton-Gorges SL, Soares BP, et al. ACR appropriateness criteria ${ }^{\oplus}$ suspected physical abuse-child. J Am Coll Radiol. 2017;14(5S):S338-S349. https://doi. org/10.1016/j.jacr.2017.01.036

7. Stavas N, Paine C, Song L, Shults J, Wood J. Impact of child abuse clinical pathways on skeletal survey performance in high-risk infants. Acad Pediatr. 2020;20(1):39-45. https://doi.org/10.1016/j.acap.2019.02.012

8. Christian CW, Levin AV; Council on Child Abuse and Neglect. The eye examination in the evaluation of child abuse. Pediatrics. 2018;142(2):e20181411. https://doi. org/10.1542/peds.2018-1411 